\title{
Electrophysiological and haemodynamic basis for application of new pacemaker technology in sick sinus syndrome and atrioventricular block ${ }^{1}$
}

\author{
RICHARD SUTTON AND PAUL CITRON \\ From Westminster Hospital, London; and Medtronic, Inc., Minneapolis, Minnesota USA
}

SUMMARY A fundamental description of pacemaker systems which are commercially available or in clinical validation is given as a background for their application in a series of 62 consecutive patients presenting over a period of 1 year for permanent cardiac pacing. The patients $(23(37 \%)$ sick sinus syndrome, $38(61 \%)$ atrioventricular block, and 1 ventricular tachycardia) were studied electrophysiologically and haemodynamically to allow the appropriate application of a pacemaker system. In sick sinus syndrome, 8 patients had permanent atrial pacing, 14 ventricular pacing, and 1 atrioventricular sequential pacing; in atrioventricular block, 8 patients had atrial synchronous ventricular inhibited pacing and the remaining 30 had ventricular pacing. A high incidence of atrial fibrillation, 9 patients, and abnormal sinus node function, 15 patients, precluded wider use of atrial synchrony. The results show benefit in acute haemodynamic studies of using systems including atrial sensing and/or pacing, and with greater availability of atrioventricular sequential and still more advanced pacemakers with dual sensing as well as dual pacing the majority of patients may be offered this benefit.

Permanent, implanted cardiac pacemakers have been used clinically since 1959 (Elmqvist and Senning, 1960). As is common with new treatments, the initial indications for use were very narrow, in this instance intended primarily for ventricular stimulation in cases of complete atrioventricular block with Adams-Stokes seizures. These early pacing systems maintained a constant regular cardiac rhythm by stimulating the ventricles asynchronously at a fixed rate, usually 70 pulses per minute. As a fuller understanding of cardiac pacing was realised, indications for use expanded to include management of symptomatic bradyarrhythmias, tachyarrhythmias, and restoration of optimal haemodynamics. As a result, the operational types of artificial pacemakers evolved so that there now exists an armamentarium of atrial and ventricular pacemaker systems, each appropriate to certain pathological situations within a broad range of cardiac diseases.

'Part of this paper was presented to the British Cardiac Society, November 1977, under the title, 'Pacing for optimal function in the spectrum of sick sinus syndrome and atrioventricular block'.

Received for publication 25 September 1978
Several atrial pacemaker systems have been available for a number of years. Atrial lead stability difficulties, particularly, have limited their use and ventricular pacemakers are generally chosen even when the disease process appears confined to the atria. Recent reports, however, cite successful long-term results with transvenous atrial leads of several designs. These include: (a) J-shaped leads which wedge within the right atrium (Fields $e t$ al., 1973), (b) smaller, tined J-shaped leads which are intended to be positioned within the right atrial appendage (Smyth et al., 1976; Kleinert et al., 1977), (c) hooking electrodes (Rosenkranz and Schaldach, 1971; Irnich et al., 1972), (d) 'screw-in' electrodes (Kleinert and Bisping, 1977), and (e) coronary sinus leads (Greenberg et al., 1978; Moss and Rivers, 1978). With the development of transvenous atrial leads which promise long-term efficacy, reassessment of the type of pacemaker system best suited to each patient is appropriate.

The object of this report is to provide a fundamental, generic description of pacemaker systems which are commercially available or in clinical validation and their indications for use, followed by clinical experience in selection and application of these systems. 


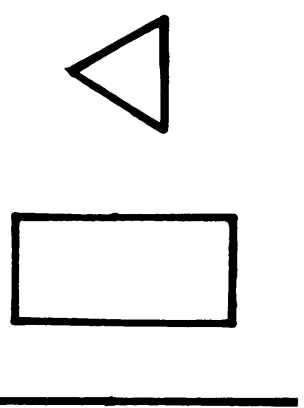

Fig. 1 A diagrammatic representation of an amplifier (triangle), oscillator (rectangle), and lead (line).

\section{Description of pacing systems}

Implantable cardiac pacemakers consist of many components including an energy source, electronic circuits, mechanical package, and heart leads. While in vivo performance characteristics rely heavily on the careful integration of all constituent parts, for present purposes we may consider the majority of available pacemakers to consist of 3 interactive functional elements: (1) the stimulation pulse forming network or oscillator; (2) the cardiac activity sensing portion or amplifier; (3) the lead system used to connect the pacemaker and the myocardium.

Fig. 1 shows the schematic representation for these elements as used in this report.

Identification of the pacing systems to be discussed is simplified considerably by the use of the generic code originally suggested by Parsonnet et al. (1974). The code consists of a sequence of three characters which describe the characteristics of the pacemaker. The first character identifies the chamber paced-A for atrium, $\mathrm{V}$ for ventricle, $\mathrm{D}$ for dual chamber pacing. The second character identifies the chamber sensed-A for atrium, $V$ for ventricle, $D$ for dual chamber sensing. The third character denotes the mode of response, if applicable-I for inhibited or blocked output in response to a sensed signal, $\mathrm{T}$ for a triggered or synchronised output. ' $O$ ' is used where the identification comment is not applicable. A VOO designation, therefore, would describe a ventricular stimulating device which does not sense intrinsic activity and thus does not modify its output in response to spontaneous activity. A VAT designation describes a ventricular stimulating pacemaker whose output is triggered by sensed atrial electrograms.

\section{(A) Asynchronous pacemaker systems}

The asynchronous pacemaker system is the most

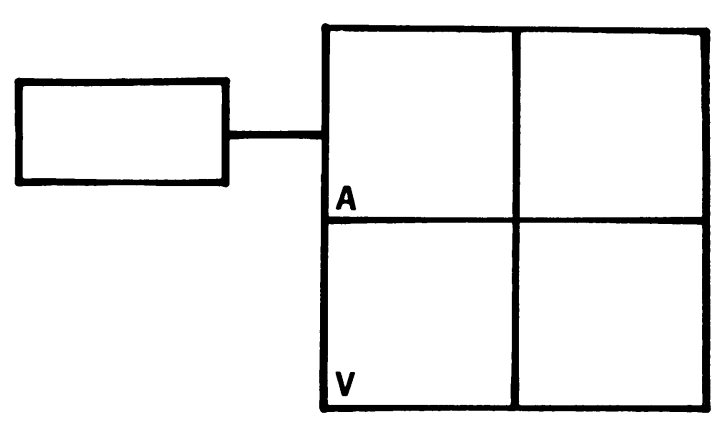

(a)

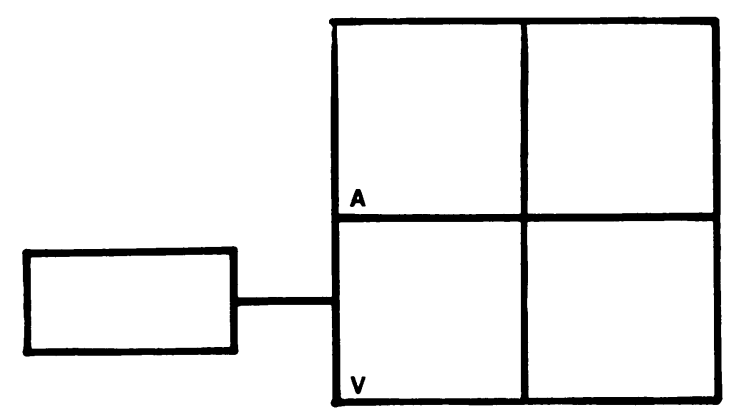

(b)

Fig. 2 (a) A four-chambered heart diagram with an oscillator connected by a lead to an atrium $(A)$;

$(V)$ denotes ventricle. This constitutes an $A O O$ pacemaker system. (b) A VOO system, an oscillator connected by a lead to a ventricle $(V)$. $(A)$ denotes atrium.

fundamental of the commercially available devices, consisting of a pulse forming network and a lead system. The purpose of this device is to provide a fixed rate train of stimuli to the atria (AOO Fig. 2a) or ventricles (VOO Fig. 2b) irrespective of and unresponsive to any intrinsic cardiac activity. Since the VOO and AOO device's output is so regular, this type of device is often incorrectly referred to as a fixed rate pacemaker instead of an asynchronous pacemaker.

While this report is primarily concerned with fully implanted pacemaker systems, it may be appropriate to digress at this point to discuss briefly radio-frequency coupled asynchronous pacing systems. These consist of a small, implanted, passive receiver/decoder to which the heart leads are connected, and an external battery-powered transmitter which is used to power and programme the receiver. Systems of this configuration received much attention in the early days of pacing as the implanted portion was significantly smaller than self-contained implanted devices because of the 
absence of batteries (Glenn et al., 1959; Cammilli et al., 1962). Consequently, these systems offered the potential of increased reliability and reduced patient exposure to repeated surgery. Improvements in implantable pacemaker design, however, obviated most of the benefits of these systems. Recently, renewed interest in these devices has been noted for the physician/patient management of episodic refractory tachycardias with rapid pacing (Iwa and Wada, 1974; Kahn et al., 1976; Waxman et al., 1978).

\section{CLINICAL APPLICATION}

\section{Atrial asynchronous pacemaker (AOO)}

Clinical use of AOO devices is rarely indicated. This type of pacemaker may find limited use in patients concurrently showing (1) stable sinus bradycardia or absolute pacemaker dependence; the latter was not seen in the present series (see below), (2) a normal His-Purkinjesy stem, and (3) improved haemodynamics with temporary atrial versus ventricular pacing. Atrial pacing is specifically contraindicated in cases of atrial paralysis (Sutton et al., 1977).

\section{Ventricular asynchronous pacemaker (VOO)}

VOO pacemakers may be indicated in patients with complete atrioventricular block, showing no intrinsic competitive rhythms and pacemaker dependence. Those with complete anterograde and retrograde atrioventricular block and sinuatrial node dysfunction manifest as sinus bradycardia, sinuatrial block, or atrial fibrillation may also be candidates for this form of pacing. The occurrence of retrograde atrioventricular atrial activation upon ventricular stimulation results in an inappropriate contraction sequence which may cause pronounced hypotension in some patients. Further, patients considered for VOO pacing should be those who would benefit little on haemodynamic grounds or because of general infirmity from more sophisticated pacemaker types. Less than 5 per cent of patients are estimated to be candidates for VOO pacemakers (Fontaine et al., 1978); one patient was found to be pacemaker dependent in this series (see below).

\section{(B) Demand pacemaker systems}

While a subset of patients exhibiting stable bradyarrhythmias such as those with complete third degree atrioventricular block exists, a majority of patients have periods of spontaneous cardiac activity during which artificial pacing may be inappropriate. Lacking any means with which to sense intrinsic cardiac activity, asynchronous pace- makers may, therefore, compete with spontaneous rhythms. Competitive pacing may produce symptomatic hypotension, ventricular tachycardia, and possibly ventricular fibrillation (Furman and Escher, 1970). By providing a properly designed cardiac electrogram sensing element (amplifier) as shown in Fig. 3a and 3b, the pacemaker can be made to be responsive to intrinsic activity. In the absence of a sufficiently rapid spontaneous cardiac rhythm, the demand pacemaker produces stimuli at a preset fixed rate (nominally $1000 \mathrm{~ms}$ to $800 \mathrm{~ms}$ interstimulus or escape interval). Electrograms detected by the amplifier modify the basic escape interval of the oscillator in one of two ways. In the case of inhibited demand devices, electrograms occurring and detected within the interstimulus interval reset the oscillator so that the next pacemaker stimulus occurs at the preset escape interval following intrinsic activity (Fig. 4). No stimuli are produced when the intrinsic rate is greater than the preset rate of the pacemaker (that is, electrogram to electrogram interval is shorter than the pacemaker escape interval). When spontaneous activity is

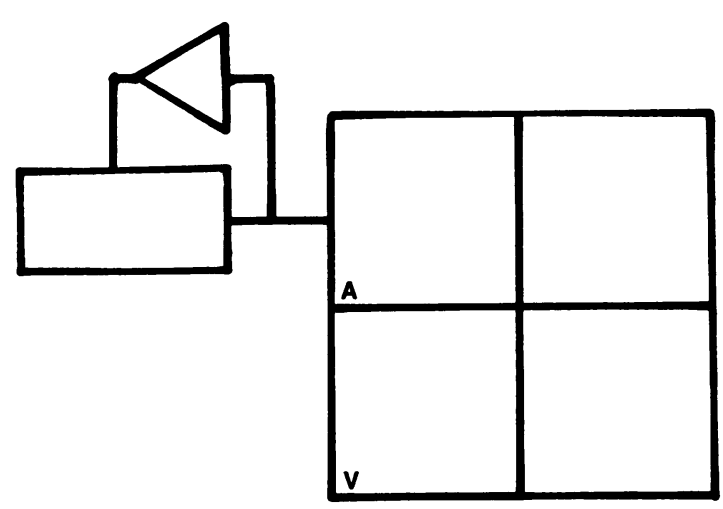

(a)



(b)

Fig. 3 (a) An atrial demand pacemaker $A A I$ or $A A T$; $(A)$ atrium, $(V)$ ventricle. (b) $A$ ventricular demand pacemaker VVI or VVT; $(A)$ atrium, $(V)$ ventricle. 


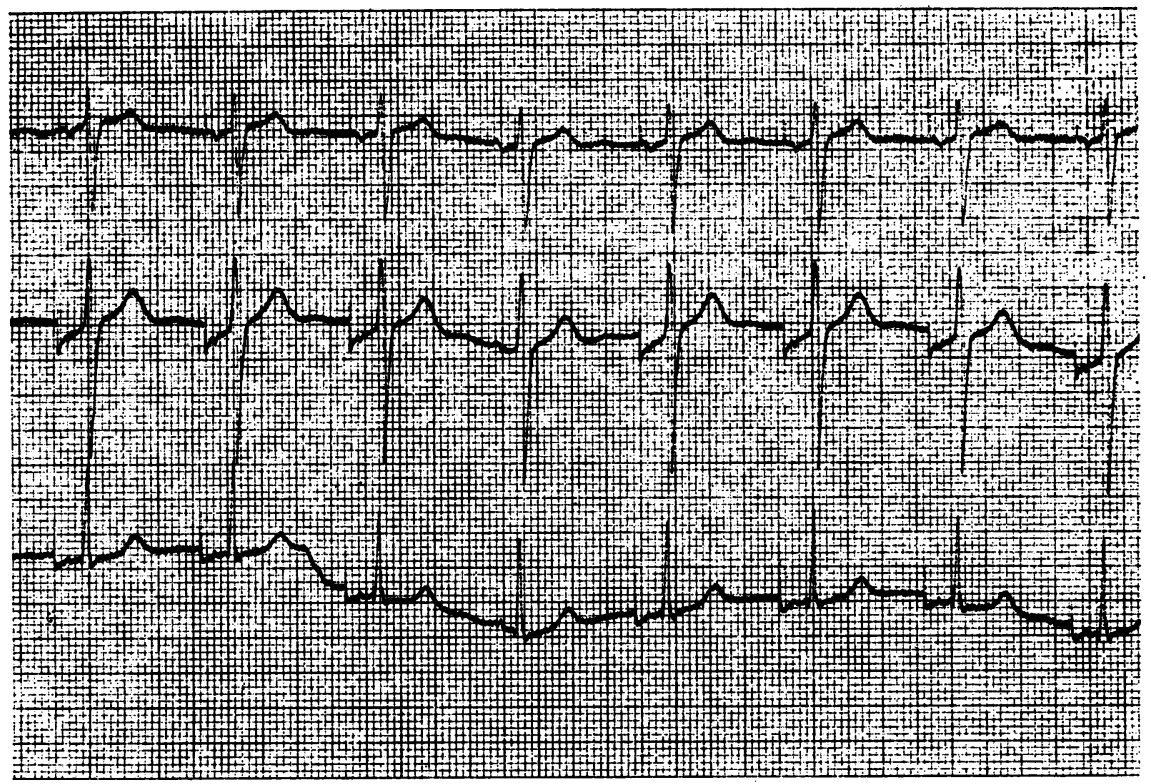

Fig. 4 A three-lead electrocardiogram. An AAI pacemaker stimulates the atrium in the first 3 beats. There is a spontaneous depolarisation at the fourth beat which inhibits the pacemaker. Atrial stimulation continues after the escape interval.

faster than the pacemaker escape interval in triggered demand pacemakers, an output stimulus occurs within the spontaneous electrogram during the absolute refractory period of the tissue (Fig. 5). Demand pacemakers of the triggered or inhibited types may be applied to either the atria (Fig. 3a)
(AAT, AAI), or to the ventricles (Fig. 3b) (VVT, VVI). While atrial and ventricular demand pacemakers are functionally identical, electrical specifications and characteristics of the sensing circuitry may be modified to accommodate the intended application (Moss, 1975).

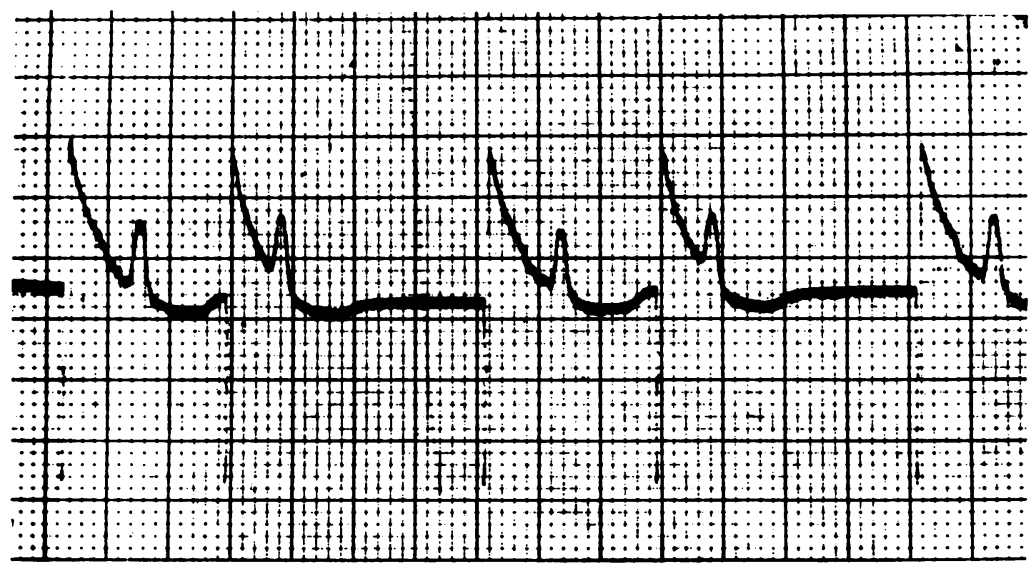

Fig. 5 An electrocardiogram of an $A A T$ pacemaker. The first, third, and fifth are pacemaker induced atrial beats. The second and fourth are spontaneous atrial beats which illustrate the triggered mode. Note the pacemaker-induced beats have a stimulus to QRS interval of $200 \mathrm{~ms}$ while the spontaneous complexes have a stimulus to QRS interval of $120 \mathrm{~ms}$. 


\section{CLINICAL APPLICATION}

(1) Atrial demand pacemakers ( $A A I, A A T$ )

Pacemakers of this type are indicated in sick sinus syndrome ${ }^{1}$ with no attendant His-Purkinje disease where haemodynamics are improved by the atrial contribution to cardiac output versus ventricular pacing (Benchimol et al., 1965; Samet et al., 1966; Befeler et al., 1971). During episodes of atrial tachycardia, these devices are ineffective, the AAI being inhibited by the rapid rate and, correspondingly, the AAT pacemaker discharging ineffectively in the $P$ wave. Since these tachycardias represent escape rhythms consequent upon episodes of sinus bradycardia or sinuatrial block (Haft, 1974), prevention of slow rhythms by the pacemaker with administration of antiarrhythmic agents often results in reducing the incidence of atrial tachycardia (Moss and Davis, 1974). Particular improvement in haemodynamic performance with atrial pacing may be obtained in cases when ventricular pacing results in retrograde atrioventricular conduction (Furman, 1973). Atrial pacing is contraindicated in atrial paralysis (Sutton et al., 1978).

(2) Ventricular demand pacemakers (VVI, VVT) Ventricular demand pacemakers are indicated in complete atrioventricular block where periods of normal conduction or spontaneous ventricular rhythms may exist and where the haemodynamic benefit of atrial systole is judged to be unnecessary,

\footnotetext{
${ }^{1}$ A single, generally accepted description of sick sinus syndrome does not exist at present. In this text sick sinus syndrome is defined as a disease of the specialised conducting system of the heart which is manifest primarily in abnormal discharge of the sinuatrial node resulting in sinus bradycardia $(<60 /$ min) and sinuatrial block. Consequent upon the bradyarrhythmias are frequent escape rhythms, some of which are tachycardiac, including atrial tachycardia, flutter, and fibrillation. Frequently the ventricular conduction system is partially involved in the disease process. The patient may present with syncope, palpitation, or dyspnoea as specific symptoms. Syncopal episodes may be typical Adams-Stokes seizures or attacks of longer duration which may simulate variants of epilepsy and transient cerebral ischaemic episodes.
}

either on the grounds of no improvement in cardiac function or where the patient is dominantly in sinus rhythm without severe delay in ventricular conduction (Paeprer et al., 1977). Use in sick sinus syndrome may be indicated where there is no retrograde atrioventricular conduction with ventricular stimulation, and where there is no clinically significant haemodynamic benefit to be obtained with more sophisticated atrioventricular pacing systems, and when atrial fibrillation is the dominant atrial rhythm. Demand systems, in particular the VVI, are indicated because patients with atrial fibrillation may manifest faster rhythms than those of the pacemaker in response to physiological situations such as exercise.

\section{(C) Atrial synchronous pacemaker systems}

During periods of stimulation using asynchronous or demand ventricular devices (VOO, VVT, VVI) in the absence of retrograde atrioventricular conduction or atrial fibrillation, atrioventricular dissociation exists (Fig. 6). In the majority of patients with normal sinus node function treated with ventricularly programmed pacemakers, the cardiac output loss resulting from atrioventricular dissociation does not manifest itself clinically. However, the clinical course in patients with a limited ability to augment ventricular stroke volume in response to physical activity, or those with ventricular pacemaker induced tachyarrhythmias (Kastor and Leinbach, 1973; Waxman and Wald, 1977), may be much improved by synchronising ventricular stimulation to atrial systole, thereby taking advantage of the atrial contribution to cardiac output and physiological heart rate change (Nathan et al., 1963; Samet et al., 1965; Karlöf, 1975). The atrial ( $P$ wave) synchronous pacemaker (VAT) consists of an atrial sensing amplifier and a ventricular stimulating oscillator arranged and connected to the heart as shown in Fig. 7. Each time an atrial electrogram is detected, the ventricular oscillator is triggered, producing a stimulus

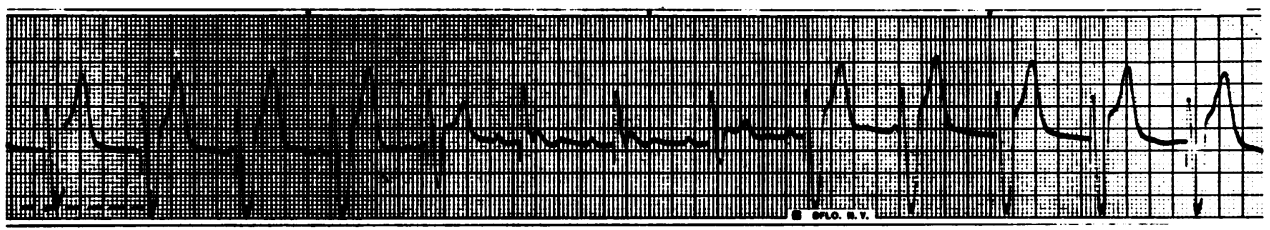

Fig. 6 A VVT pacemaker electrocardiogram. Atrioventricular dissociation exists during the first 4 complexes followed by a fusion beat, 3 sinus conducted beats on which a pacemaker stimulus falls in the absolute refractory period, and the strip ends with 5 complexes of ventricular pacing with atrioventricular dissociation. 


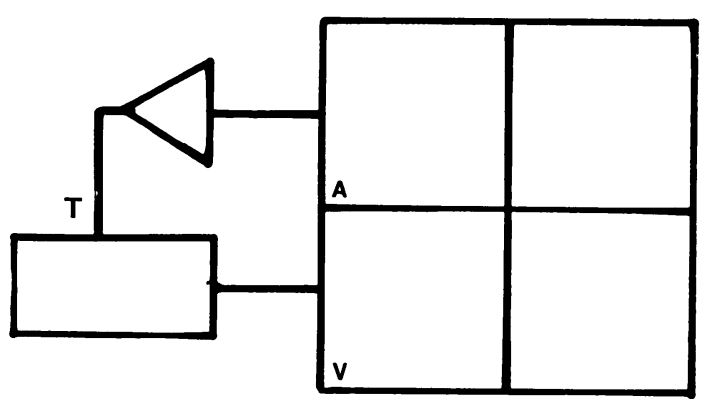

Fig. 7 A VAT pacemaker. (A) atrium, $(V)$ veritricle, (T) triggering amplifier.

after an interval which mimics the normal atroventricular delay interval (Fig. 8). In the absence of sensed atrial electrograms which may result from atrial lead displacement, during atrial fibrillation or sinus arrest, the atrial synchronous pacemaker operates as an asynchronous ventricular pacemaker (VOO) at a preset basic rate. A significant shortcoming of atrial synchronous devices (VAT) is their inability to sense and respond to spontaneous ventricular activity. Several atrial synchronous pacemaker-induced arrhythmias have been shown and suggested (Castellanos et al., 1968).

An evolutionary version of the atrial synchronous

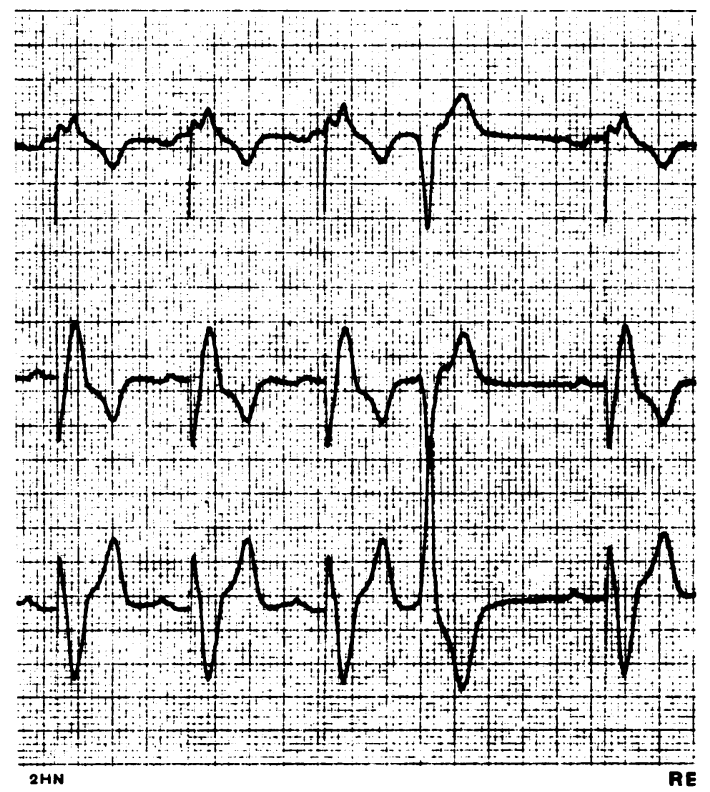

Fig. 8 A 3-lead electrocardiogram of an atrial synchronous pacemaker. The first 3 beats show atrial synchrony inhibition of the ventricular oscillator. The last beat shows atrial synchrony (VD $\frac{T}{I}$ pacemaker).

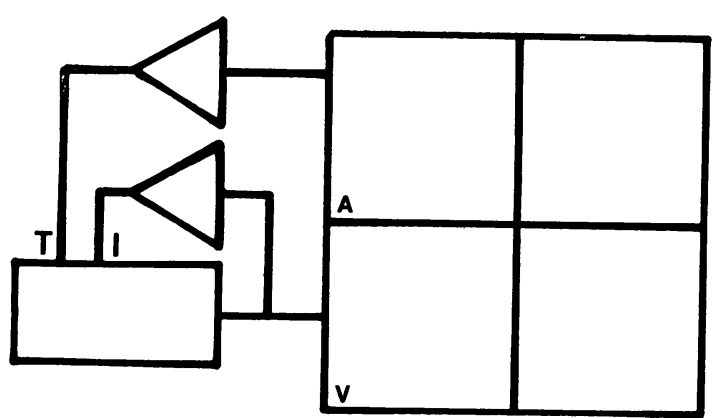

Fig. 9 An atrial synchronous ventricular inhibited pacemaker VD $\frac{T}{I} \cdot(A)$ atrium, $(V)$ ventricle,

$(T)$ triggering amplifier, (I) inhibiting amplifier.

device (VAT) which offers a potential for minimising previously reported induced arrhythmias is shown in Fig. 9. In addition to the functional elements of the VAT pacemaker, this device VD $\frac{T^{1}}{\mathrm{I}}$ has a second sensing amplifier intended to sense intrinsic ventricular activity and inhibit the ventricular oscillator when necessary. In the absence of sensed atrial electrograms, the VD $\frac{T}{\mathrm{I}}$ functions as an inhibited ventricular demand pacemaker (VVI). During periods of normal sinus rhythm, without normal ventricular response, this device behaves as a VAT pacemaker. When normally conducted ventricular complexes or ventricular ectopic activity occurs, the VD $\frac{T}{\mathrm{I}}$ 's oscillator is inhibited for that cycle in a manner similar to a VVI device.

\section{CLINICAL APPLICATION}

Atrial synchronous pacemakers (VAT, VD $\frac{\mathrm{T}}{\mathrm{I}}$ )

Atrial synchronous pacemakers are indicated in complete or intermittent heart block with normal sinus node function where the haemodynamic benefit of the properly timed atrial systole is necessary or worth while (Karlöf, 1975). These patients should show atrioventricular block frequently and are most suitable if atrioventricular block develops on exercise. Atrial synchronous devices are contraindicated in sick sinus syndrome or atrial fibrillation. Excessively late retrograde atrial activation upon ventricular stimulation may

${ }^{1}$ Note: The third symbol of the generic nomenclature code has been modified to include more advanced pacemakers. 


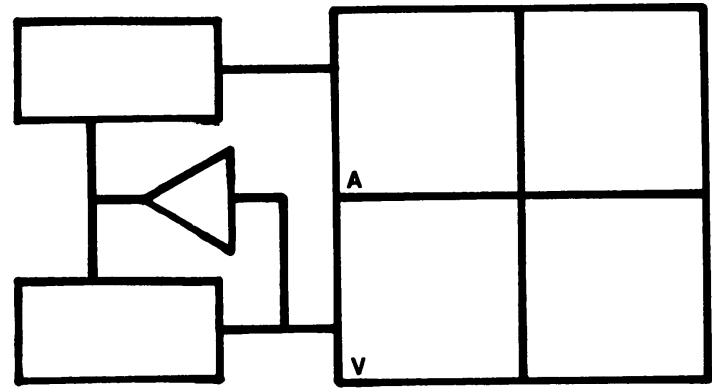

Fig. 10 An atrioventricular sequential pacemaker DVI. (A) atrium, $(V)$ ventricle.

result in pacemaker-induced tachycardia and, therefore, the suitability of specific pacemaker designs must be assessed in patients with retrograde conduction.

\section{(D) Atrioventricular sequential pacemakers}

The VAT type device maintains atrioventricular association in patients with normal sinus rhythms within the operational specifications of the pacemaker. Maintenance of the atrioventricular contraction sequence in patients with sinus node dysfunction, primarily bradycardias, may be accomplished with a DVI pacemaker as shown in Fig. 10. Both oscillators in this pacemaker configuration are inhibited by the ventricular electrogram only. If no ventricular activity is sensed within the escape interval of the atrial oscillator, a stimulus is delivered to the atria. If ventricular activity is then not sensed within a preset atrioventricular delay interval, the ventricular oscillator produces a ventricular stimulus. Conducted ventricular beats or ectopic ventricular activity inhibit and reset both oscillators (Fig. 11).

\section{CLINICAL APPLICATION}

\section{Atrioventricular sequential pacemaker (DVI)}

DVI pacemakers are indicated in sick sinus syndrome with accompanying His-Purkinje disease or in atrioventricular block with accompanying evidence of abnormal sinus node function where haemodynamic benefit from correctly placed atrial systole is shown. Further, DVI pacing may have application in the overdrive of atrial and ventricular ectopic rhythms and re-entry tachyarrhythmias (Fields et al., 1973).

\section{Patients and methods}

Since many of the barriers to using atrial systems have been effectively overcome, 62 consecutive patients presenting for permanent pacing at Westminster Hospital, London, between February 1977 and February 1978, have been studied for selection of optimal pacing system. There were 40 men and 22 women, mean age was 64 years (range 51 to 89). Twenty-three patients who were syncopal or near syncopal had sick sinus syndrome diagnosed on routine electrocardiography and/or Holter monitoring and 38 had atrioventricular block with syncopal episodes or heart failure. One was given ventricular pacing for overdrive

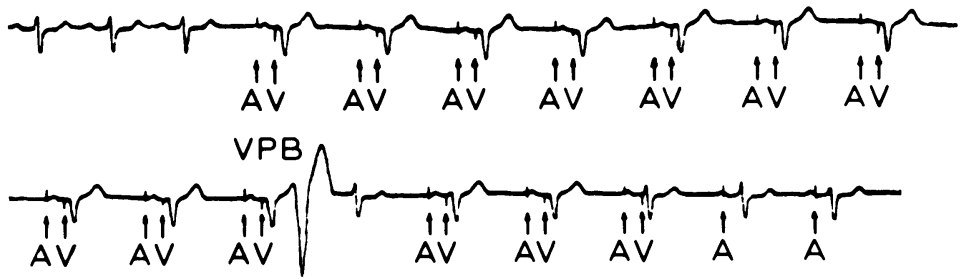

Fig. 11 An electrocardiogram of an atrioventricular sequential pacemaker DVI. The 2 strips are not continuous. The first 3 complexes in the upper trace show sinus rhythm without atrioventricular block, both oscillators are inhibited; the remaining complexes show atrioventricular sequential pacing. The lower trace shows atrioventricular sequential pacing in the first 3 complexes. There then follows a ventricular premature beat (VPB) which inhibits both oscillators; the next beat is sinus conducted which also inhibits both oscillators. The sixth and seventh complexes show atrioventricular sequential stimulation and the eighth complex shows atrial stimulation and ventricular fusion between normal conduction and ventricular stimulation. The ninth complex shows inhibition of the ventricular oscillator with the atrial stimulus falling in the atrial absolute refractory period. (A) Atrial stimulus, $(V)$ ventricular stimulus. 
of ventricular tachycardia. All patients underwent a 24-hour period of Holter monitoring and an exercise stress (submaximal) using a bicycle ergometer. In the patients with sick sinus syndrome and with atrioventricular block, sinus node recovery time and His bundle electrograms were recorded, omitting those with atrial fibrillation. A haemodynamic study was performed on all patients except those with atrial fibrillation and those with atrioventricular block in whom abnormal sinuatrial function was shown. Measurements included pulmonary capillary wedge, pulmonary artery, and aortic pressures, and cardiac output measured by the thermodilution technique. These were recorded during the patient's own rhythm, which was sinus bradycardia (in sick sinus syndrome) or complete atrioventricular block, during ventricular pacing at $70 / \mathrm{min}$, and during either atrial pacing at $70 / \mathrm{min}$ for patients with sick sinus syndrome or atrioventricular pacing (DVI) for patients with atrioventricular block. After the haemodynamic study the sinus node recovery time was repeated after administering atropine $1.2 \mathrm{mg}$ intravenously. In cases of atrial fibrillation and when sinuatrial node function was abnormal in the atrioventricular block group, the patients proceeded directly to permanent ventricular pacing. The latter group would now receive a DVI pacemaker, but during this series supplies of this unit were not freely available. During ventricular pacing absence of retrograde atrioventricular conduction was shown using an atrial electrogram.

The results of these physiological studies were used to select the type of pacemaker for permanent implantation.

\section{Results of physiological studies}

Of the 23 patients with sick sinus syndrome, who constituted 37 per cent of the whole group, 8 displayed optimal haemodynamics and were suitable electrophysiologically for atrial pacing using a rate adjustable AAI (Medtronic 5963A) or a non-rate adjustable AAI (Medtronic 5951AD) pacemaker. Fourteen had no haemodynamic benefit from atrial pacing or were either electrophysiologically unsuitable for atrial pacing or showed atrial fibrillation as their dominant rhythm. They received permanent VVI ventricular stimulators (Medtronic 5973; Telectronics P150B, CPI 0502, 0505; Vitatron C2120). One patient (case 5, Table 1) required atrial pacing on the basis of the haemodynamics but showed severe His-Purkinje system disease also and this required DVI atrioventricular sequential pacing (Medtronic 5992). The electrophysiological results are shown in Table 1. The

Table 1 Electrophysiological and haemodynamic data in sick sinus syndrome

\begin{tabular}{|c|c|c|c|c|c|c|c|}
\hline \multirow[t]{2}{*}{ Case no. } & \multirow[t]{2}{*}{ SNRT } & \multirow[t]{2}{*}{$H V$} & \multirow{2}{*}{$\begin{array}{l}\text { Retrograde } \\
\text { atrioventricular } \\
\text { conduction }\end{array}$} & \multicolumn{3}{|c|}{ Cardiac output (l/min) } & \multirow[t]{2}{*}{ Pacer type } \\
\hline & & & & Own rhythm & $\begin{array}{l}\text { Atrial pacing } \\
(70 / \text { min })\end{array}$ & $\begin{array}{l}\text { Ventricular } \\
\text { pacing } \\
(70 / \text { min })\end{array}$ & \\
\hline 1 & Abnormal & Normal & + & $3 \cdot 86$ & $4 \cdot 42$ & $3 \cdot 25$ & AAI \\
\hline 2 & Abnormal & Normal & + & $3 \cdot 67$ & - & $3 \cdot 43$ & VVI \\
\hline 3 & Abnormal & Normal & + & $4 \cdot 66$ & $5 \cdot 47$ & $3 \cdot 38$ & AAI \\
\hline 5 & Abnormal & Abnormal & 0 & $4 \cdot 01$ & $3 \cdot 55^{\star}$ & $2 \cdot 63$ & DVI \\
\hline 6 & Abnormal & Normal & + & $4 \cdot 18$ & $5 \cdot 26$ & $4 \cdot 08$ & AAI \\
\hline 7 & Abnormal & Normal & + & - & - & - & VVI \\
\hline 8 & Abnormal & Normal & + & $2 \cdot 87$ & $2 \cdot 51$ & $2 \cdot 75$ & AAI \\
\hline 9 & Abnormal & Normal & + & $5 \cdot 75$ & $6 \cdot 80$ & $6 \cdot 02$ & AAI \\
\hline 10 & Abnormal & Normal & + & $2 \cdot 60$ & 3.90 & $3 \cdot 19$ & VVI \\
\hline 11 & $\mathrm{AF}$ & Normal & 0 & 一 & - & - & VVI \\
\hline 12 & AF & Normal & 0 & - & - & - & VVI \\
\hline 13 & Abnormal & Normal & + & $4 \cdot 47$ & $5 \cdot 18$ & $4 \cdot 38$ & AAI \\
\hline 15 & Abnormal & Normal & 0 & $7 \cdot 37$ & $5 \cdot 42$ & $5 \cdot 51$ & VVI \\
\hline 16 & AF & Normal & 0 & - & - & - & VVI \\
\hline 17 & Abnormal & Normalt & + & - & - & - & VVI \\
\hline 18 & Abnormal & Normal & 0 & $4 \cdot 42$ & $4 \cdot 28$ & 3.95 & VVI \\
\hline 19 & AF & Normal & 0 & - & - & - & VVI \\
\hline 20 & Abnormal & Normal & + & 3.59 & $3 \cdot 76$ & $3 \cdot 22$ & AAI \\
\hline 21 & Abnormal & Normal & + & $3 \cdot 43$ & 3.61 & 3.00 & AAI \\
\hline 22 & Abnormal & Normal & + & $4 \cdot 18$ & $4 \cdot 77$ & $3 \cdot 24$ & AAI \\
\hline 23 & Abnormalł & Normal & + & $7 \cdot 92$ & $7 \cdot 18$ & $6 \cdot 27$ & VVI \\
\hline
\end{tabular}

^DVI pacing at $70 / \mathrm{min}$.

†Bifascicular block on scalar electrocardiogram.

¥Sinus rhythm consistently greater than $70 / \mathrm{min}$.

SNRT Sinus node recovery time; AF, atrial fibrillation. 
sinus node recovery time was more than 150 per cent of control at switch-off from atrial pacing at $140 \mathrm{ppm}$ in all patients before and after atropine. Low right atrium (A) to His time was less than $200 \mathrm{~ms}$ in all patients.

The His to ventricular time was between 35 and $55 \mathrm{~ms}$ in all except case 4 where it was $60 \mathrm{~ms}$, and case 5 where it was $85 \mathrm{~ms}$. The presence of retrograde atrioventricular conduction was variable but, when present, was one feature in determining the need for atrial rather than ventricular permanent pacing.

The haemodynamic results in 16 of these 23 patients are presented also in Table 1 . Only the cardiac outputs are shown. The type of pacemaker implanted is indicated in the right hand column of the figure. Cases 2 and 4 were studied early in the series when the chief criterion for ventricular pacing was that no fall in output occurred between the patient's own rhythm and ventricular pacing. As this was not the case in these 2 patients, atrial pacing outputs were not performed. The choice of pacemaker in case 5 was discussed above. Generally atrial pacing was shown to have an advantage over ventricular pacing. These improvements did not reach statistical significance; however, when left ventricular filling was also taken into account and ventricular function curves were plotted a statistically significant $(P<0.05)$ improvement of atrial over ventricular pacing was found (Sutton and Perrins, 1979). Only cases 8 and 15 showed no improvement with atrial pacing over ventricular pacing; in retrospect, cases 2 and 4 may have derived benefit from atrial pacing rather than ventricular. Case 7 was not studied haemodynamically because atrial pacing for sinus node recovery time caused an Adams-Stokes attack. Resuscitation was accomplished by instituting ventricular pacing immediately. Case 10 was considered most suitable for atrial pacing; however, atrial thresholds in excess of $4 \mathrm{~V}$ at $1.0 \mathrm{~ms}$ were found in the right atrial appendage. Therefore, ventricular pacing was used. Cases $11,12,14,16$, and 19 showed atrial fibrillation as their dominant rhythm and were for this reason not studied haemodynamically. Case 17 was given ventricular pacing because of the existence of bifascicular block (right bundle-branch block and left anterior hemiblock) despite a normal His-ventricular time. Case 23 showed better cardiac output with atrial pacing than ventricular but because his dominant rhythm was sinus at a rate of greater than $70 \mathrm{ppm}$, ventricular pacing was chosen. Ventricular pacing in sick sinus syndrome can be associated with severe haemodynamic changes (Blömer et al., 1977; Patel et al., 1977). Arterial pressure may fall at the onset of ventricular pacing taking over from sinus rhythm, a finding also made in patients with atrioventricular block. The latter patients usually recover their arterial pressures within a few cardiac cycles. However, in sick sinus syndrome, recovery may be incomplete or not exist and falls of 70 or more $\mathrm{mmHg}$ in arterial pressure may be seen. Appropriately timed retrograde atrioventricular conduction is probably the major factor and when the pulmonary capillary wedge pressure is observed during retrograde atrioventricular conduction a high systolic wave can be observed. This is, of course, superimposition of the ' $a$ ' wave generated by the retrograde atrioventricular conduction upon the normal ' $v$ ' wave occurring at that time.

Two patients before this series experienced Adams-Stokes attacks during ventricular pacing which was shown to be the result of retrograde atrioventricular conduction causing hypotension. In each case normal ventricular pacing was observed during the Adams-Stokes attacks. Both patients were converted to atrial pacing. In the first, complete abolition of attacks was achieved; in the second, control was obtained using a temporary atrial lead. This lead subsequently displaced with loss of atrial pacing and an Adams-Stokes attack. Resuscitation was unsuccessful. Two patients in this series (cases 2 and 17) also experienced episodes of severe hypotension during retrograde atrioventricular conduction. In case 2 a coincident atrioventricular bypass tract was considered responsible (Waxman and Wald, 1977). Control was obtained by use of a beta-blocking drug. Case 17 had retrograde atrioventricular conduction blocked by a betablocking drug with symptomatic relief. Both of these patients would now be considered for a DVI pacemaker (Fields et al., 1973).

Of the 38 patients with atrioventricular block, comprising 61 per cent of the whole group, only 10 patients had normal sinuatrial node function. Of these, 8 were fitted with atrial synchronous ventricular inhibited VD $\frac{T}{\mathrm{I}}$ pacemakers (Medtronic

5993) using an endocardial right atrial lead for sensing atrial activity (Medtronic 6991-7 patients and transvenous endocardial screw-in lead Medtronic SP0039BV-one patient). The remaining 2 were in dominant sinus rhythm with rare episodes of atrioventricular block which was specifically not induced by exercise. Atrial fibrillation was either permanent or intermittent in 9 patients and at study a further 15 patients showed evidence of abnormal sinuatrial node function either on routine electrocardiography, Holter monitoring, exercise, or abnormal sinus node recovery times, or a com- 
Table 2 Haemodynamic data in atrioventricular block

\begin{tabular}{llll}
\hline Case no. & Own rhythm & $\begin{array}{l}\text { CO l/min } \\
\text { VP 70/min }\end{array}$ & DVI* 70/min \\
\hline 1 & & 3.76 & 5.07 \\
2 & 4.09 & 4.54 & 4.32 \\
3 & 3.94 & 2.98 & 3.60 \\
4 & 4.02 & 5.09 & 5.61 \\
5 & 5.93 & 3.62 & 4.27 \\
6 & 3.62 & 4.20 & 4.95 \\
7 & 3.35 & 3.51 & 4.23 \\
8 & 4.01 & 4.26 & 5.42 \\
\hline
\end{tabular}

^External VD $\frac{T}{\mathrm{I}}$ was not available for these studies. Therefore, an external DVI was used with fixed PR $0.20 \mathrm{~ms}$.

bination of these. One patient showed retrograde atrioventricular conduction which constituted a contraindication to use of an atrial synchronous pacemaker. Three patients were not studied because of general frailty or other complicating conditions. Thus 74 per cent of patients with atrioventricular block were unsuitable for atrial synchronous pacing. All these patients received ventricular stimulators; in retrospect, some of these may have received benefit from DVI atrioventricular pacing. The haemodynamic results of the 8 patients with normal sinuatrial node functions are shown in Table 2. The second patient showed little benefit from atrial systole at rest. However, his exercise performance was greatly increased after atrial synchronisation. The exercise studies were made separately without haemodynamic measurements. At left ventricular angiography his ejection fraction was 20 per cent with generally impaired wall motion and his coronary arteries were normal. The inferred aetiology of his conduction tissue disease is, therefore, cardiomyopathy. Case 4 was in sinus rhythm at the time of study. It is considered that this offers an explanation of his failure to show any significant difference in cardiac output in the 3 modes studied.

Atrial synchronisation was shown to give a statistically significant improvement over ventricular pacing $(P>0.05)$ in these 8 patients (Sutton and Perrins, 1979).

\section{Discussion}

This paper has presented an explanation of available pacing systems illustrating their functional simplicity by means of block diagrams. Indications and contraindications to each system have been given, but in some aspects there remains controversy (Table 3 ).

Table 3 Indications and contraindications for available and advanced pacemaker systems

\begin{tabular}{|c|c|c|}
\hline Pacer & Indications & Contraindications \\
\hline AOO & $\begin{array}{l}\text { Severe sinus bradycardia without physiological } \\
\text { rate increase }\end{array}$ & $\begin{array}{l}\text { Atrial paralysis } \\
\text { High atrial threshold } \\
\text { More rapid atrial rates resulting in competitive } \\
\text { stimulation }\end{array}$ \\
\hline VOO & Atrioventricular block with pacemaker dependence & $\begin{array}{l}\text { Intermittent atrioventricular block } \\
\text { Ventricular extrasystoles }\end{array}$ \\
\hline AAI/AAT & $\begin{array}{l}\text { Sick sinus syndrome with or without retrograde } \\
\text { atrioventricular conduction }\end{array}$ & $\begin{array}{l}\text { Atrial paralysis } \\
\text { High atrial threshold } \\
\text { Sick sinus syndrome with His-Purkinje disease } \\
\quad \text { (atrioventricular block) }\end{array}$ \\
\hline VVI/VVT & $\begin{array}{l}\text { Atrioventricular block with atrial fibrillation } \\
\text { Sick sinus syndrome without retrograde conduction } \\
\text { Sick sinus syndrome with atrial fibrillation } \\
\text { Sick sinus syndrome with no haemodynamic benefit of } \\
\text { atrial pacing }\end{array}$ & $\begin{array}{l}\text { Poor haemodynamic response to VVI or VVT because } \\
\text { of induced atrioventricular dissociation }\end{array}$ \\
\hline VAT & $\begin{array}{l}\text { Atrioventricular block with normal sinus node } \\
\text { function }\end{array}$ & $\begin{array}{l}\text { Atrioventricular block with abnormal sinus node } \\
\text { function }\end{array}$ \\
\hline VD $\frac{T}{\mathbf{I}}$ & , & Atrioventricular block with atrial fibrillation \\
\hline DVI & $\begin{array}{l}\text { Atrioventricular block with abnormal sinus node } \\
\text { function } \\
\text { Sick sinus syndrome with atrioventricular block }\end{array}$ & $\begin{array}{l}\text { Atrioventricular block or sick sinus syndrome with } \\
\text { atrial fibrillation }\end{array}$ \\
\hline $\mathrm{DD} \frac{\mathrm{TI}}{\mathrm{I}}$ & $\begin{array}{l}\text { Atrioventricular block with abnormal sinus node } \\
\text { function } \\
\text { Sick sinus syndrome with atrioventricular block } \\
\text { Atrioventricular block with normal sinus node function }\end{array}$ & $\begin{array}{l}\text { Atrioventricular block or sick sinus syndrome with } \\
\text { atrial fibrillation }\end{array}$ \\
\hline
\end{tabular}

Definition of symbols is given in the text. 
Therefore, the actual clinical use in one pacemaker centre has been cited.

Choice of atrial or ventricular pacing in sick sinus syndrome is one area of controversy. This paper has shown that electrophysiological and haemodynamic considerations are necessary in making this choice. Haemodynamic benefit is usually available to the patient by using atrial or atrioventricular pacing; however, at present there is no available means of synchronising atrial stimulation rate with the body's needs so as to increase the pacing rate on exercise, for instance. This should produce greater haemodynamic benefit. Ventricular pacing can have undesirable effects by inducing retrograde atrioventricular conduction. Despite the fact that these two problems favour atrial pacing, this mode remains contraindicated in atrial fibrillation and atrial paralysis and when there is significant His-Purkinje system disease.

The onset of atrial fibrillation in the sick sinus syndrome has been shown to improve symptoms and especially to avert syncopal episodes (Vera et al., 1977). Our patients with atrial fibrillation were all new cases who presented because of syncopal episodes despite the rhythm, so atrial fibrillation is not a panacea for syncope in this syndrome. Adoption of atrial pacing has been hindered by the expectation that atrioventricular block would develop in the foreseeable future, necessitating a premature change in the pacemaker system. Available data in comparatively large series of permanent atrial pacing, however, point against this (Kleinert et al., 1977; Citron et al., 1978; Greenberg et al., 1978). Despite the fact that the majority of these patients did not undergo detailed electrophysiological study before the choice of atrial pacing (Kleinert et al., 1977; Citron et al., 1978), it appears that valuable guidance may be gained about the possible future development of atrioventricular block (Narula, 1971; Rosen et al., 1971). Other contraindications to atrial pacing have been seen in this series whose small size may not therefore show the true incidence of high atrial threshold and the presentation of sick sinus syndrome with syncope caused by sinuatrial block but intrinsically fast sinus rate at other times (one case of each here). These two conditions are further contraindications to atrial pacing. At present, approximately one-third of patients with sick sinus syndrome will be suitable for atrial pacing. With increased availability of DVI and more advanced pacemakers which offer sensing and pacing in both atrium and ventricle (Fig. 12), all patients can receive atrial as weil as ventricular leads except when atrial fibrillation, atrial paralysis, or high atrial threshold exists. When atrial stimulation is followed by a normal or a stimulated ventricular complex, retrograde atrioventricular conduction is minimised. It may be necessary to keep the PR interval short to prevent re-entry mechanisms (Fields et al., 1973). Use of dual chamber pacemakers allays anxiety about the onset of atrioventricular block with atrial pacing.

Atrial pacing may have two further advantages in suitable patients. The incidence of escape tachycardias may be reduced or abolished even without the use of antiarrhythmic drugs and there may be a reduced rate of systemic embolism which has been claimed to be high, both without pacing and with ventricular pacing (British Medical fournal, 1973).

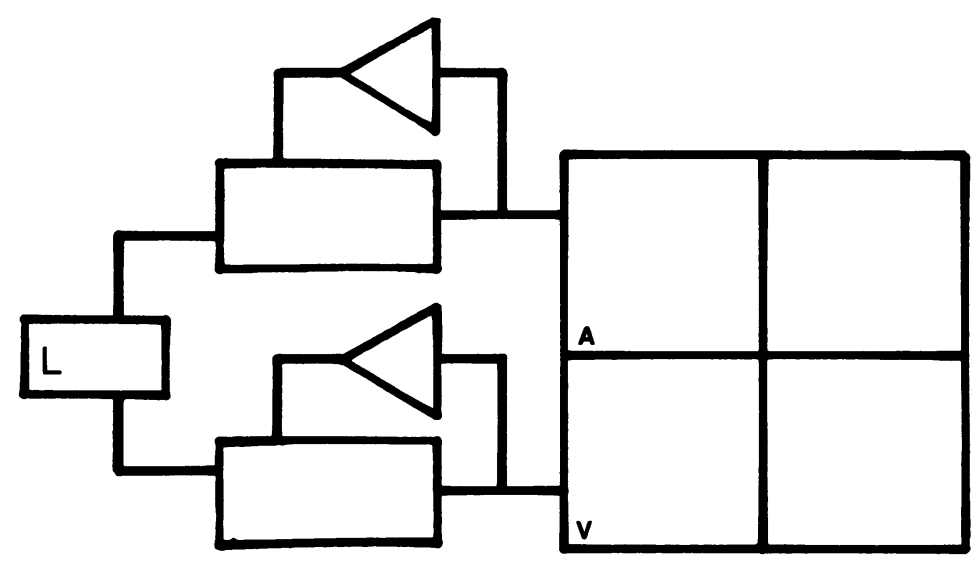

Fig. 12 Advanced pacemaker DDT $\frac{T I}{I}$ which senses and paces in both atrium and ventricle, the co-ordination by means of a logic circuit $L$. $(A)$ atrium, $(V)$ ventricle. 
The indications for addition of atrial synchrony to ventricular pacing in atrioventricular block remain unclear. The lack of clarity has been dominated by difficulties with endocardial atrial leads often necessitating epicardial lead placement and by the relatively short life characteristics of early atrial synchronous pacemakers. A more encouraging report has recently been made by Carlens et al. (1977) using epicardial atrial lead placement by mediastinoscopy. This technique has not, however, achieved wide acceptance. For atrial synchronous ventricular pacing to achieve wide application, a satisfactory all endocardial lead system is necessary. Reports of large series using atrial leads with passive fixation mechanisms (Kleinert et al., 1977; Greenberg et al., 1978) indicate that this is now possible. With modern low current drain circuits, atrial synchronous pacemakers can be expected to exhibit much better life characteristics. Now the chief determinant of life will be the proportion of time during which the ventricles are driven at higher rates than $70 \mathrm{ppm}$. In this context a fresh review of patients presenting with atrioventricular block is justified.

Studies of sinuatrial node function by Holter monitoring, exercise stress test, and sinus node recovery time in patients with atrioventricular block in this series have shown a high incidence of abnormal sinus node function. There has also been a high incidence of intermittent or permanent atrial fibrillation. Both of these conditions are contraindications to atrial synchronous pacing. In 3 patients, prepacing studies were contraindicated because of frailty or complicating conditions. Thus atrial synchronous pacing was also contraindicated. One patient showed complete anterograde atrioventricular block but retrograde atrioventricular conduction was preserved. This may lead to atrial synchronous pacemaker-induced tachycardias (Castellanos et al., 1968). In total, $28(74 \%)$ patients were unsuitable for atrial synchronous pacemakers; however, the 15 with abnormal sinus node function and the patient with retrograde atrioventricular conduction should now be considered for DVI atrioventricular sequential pacemakers. Two patients with normal sinus node function had rare syncopal episodes, and atrioventricular block was not induced on exercise. Atrial synchronous pacemakers were not considered an advantage in these cases and ventricular demand VVI pacemakers were implanted.

The high incidence of abnormal sinus node function in patients with atrioventricular block was unexpected but is considered to be a manifestation of conduction tissue disease occurring throughout the heart. Atrial fibrillation is often the final rhythm in patients with sick sinus syndrome (Vera et al., 1977) and atrial fibrillation may be seen to develop during the course of ventricular pacing for atrioventricular block. Shaw and Eraut (1970) have indicated that atrial fibrillation in atrioventricular block patients carries a poor prognosis. It is possible to postulate that many patients with atrioventricular block develop abnormal sinus node function and evolve towards atrial fibrillation as their conduction tissue disease increases. When an atrial synchronous pacemaker is replaced it is suggested that sinus node function is reassessed because atrioventricular sequential pacemaker DVI may have become more appropriate. Rapid technological development has resulted in the need for modification to the Parsonnet et al. (1974) classification of pacemakers. We propose that the third letter, which applies to the chamber used for sensing or triggering, be fractionated so that the activity of the atrium in this respect becomes the numerator and the ventricle the denominator. In some cases it may be necessary to indicate that both inhibition and triggering occur from the atrium such as in an advanced pacemaker which senses and paces in the atrium but also acts as an atrial triggered (synchronous) unit. The pacemaker in Fig. 12 should be represented by the symbols DD $\frac{T I}{\mathrm{I}}$ according to our proposed modification of the Parsonnet recommendations.

\section{Conclusions}

Sick sinus syndrome and atrioventricular block represent ends of a spectrum of conduction tissue disease with considerable overlap between them. Sick sinus syndrome represents 37 per cent of patients presenting for permanent pacing with either syncope or heart failure. With the advent of an electrode yielding satisfactory endocardial sensing and pacing, a need is created for electrophysiological and haemodynamic studies to determine the optimal pacing system for each patient. In the future some patients now given ventricular pacing and atrioventricular sequential pacing will benefit from an advanced type of pacemaker which senses and paces both atrium and ventricle.

\section{References}

Befeler, B., Cohen, L. S., Hildner, F. J., Javier, R. P., Narula, O. S., and Samet, P. (1971). Atrial contribution to ventricular function in the sitting position. Chest, 60, 240-243.

Benchimol, A., Ellis, J. G., and Diamond, E. G. (1965). Haemodynamic consequences of atrial and ventricular 
pacing in patients with normal and abnormal hearts. Effects of exercise at a fixed atrial and ventricular rate. American Journal of Medicine, 39, 911-922.

Blömer, H., Wirtzfeld, A., Delius, W., and Selvening, H. (1977). Das Sinusknoten-Syndrom. Perimed Verlag Dr. med D. Straube, Erlangen.

British Medical fournal (1973). Editorial. The sick sinus syndrome, 2, 677-678.

Cammilli, L., Pozzi, R., and Drago, G. (1962). Remote heart stimulation by radio frequency for permanent rhythm control in the Morgagni-Adams-Stokes syndrome. Surgery, 52, 765-776.

Carlens, E., Ericsson, M., Levander-Lingdren, M., and Pehrsson, K. (1977). Detector electrode introduced by mediastinoscopy for atrial triggered cardiac pacing. A follow-up study of electrode function in 82 patients. British Heart fournal, 39, 1265-1272.

Castellanos, A., jun., Lemberg, L., Rodrigues-Tacker, L., and Berkovits, B. V. (1968). Atrial synchronized pacemaker arrhythmias: revisited. American Heart fournal, 76, 199-208.

Citron, P., Smyth, N. P. D., Kleinert, M., and Kahn, A. R. (1978). Clinical experience with a new transvenous lead. Chest, 73, 193-197.

Elmqvist, R., and Senning, A. (1960). An implantable pacemaker for the heart. In Medical Electronics, Proceedings of the Second International Conference on Medical Electronics, Paris, 24-27 fune, 1959, pp. 253-254, ed C. N. Smyth. Iliffe and Sons, London.

Fields, J., Berkovits, B. V., and Matloff, J. M. (1973). Surgical experience with temporary and permanent A-V sequential demand pacing. Fournal of Thoracic and Cardiovascular Surgery, 66, 865-877.

Fontaine, G., Frank, R., Vedel, J., Welti, J., and Grosogeat, Y. (1978). Clinical hazards related to double pacing systems. In Proceedings of First European Symposium on Cardiac Pacing, p. 16. Abstracts. British Pacing Group, London.

Furman, S. (1973). Therapeutic uses of atrial pacing. American Heart fournal, 86, 835-840.

Furman, S., and Escher, D. J. (1970). Non-competitive pacing: demand and ventricular synchronous. In Principles and Techniques of Cardiac Pacing, pp. 136-154. Harper and Row, New York.

Glenn, W. W. L., Mauro, A., Longo, E., Lavietes, P. H., and Mackay, F. J. (1959). Remote stimulation of the heart by radiofrequency transmission. New England fournal of Medicine, 261, 948-951.

Greenberg, P., Castellanet, M., Messenger, J., and Ellestad, M. H. (1978). Coronary sinus pacing. Circulation, 57, 98-103.

Haft, J. I. (1974). Treatment of arrhythmias by intracardiac electrical stimulation. Progress in Cardiovascular Diseases, 16, 539-568.

Irnich, W., Bleifeld, W., and Effert, S. (1972). Permanente transvenöse Elekrostimulation des Herzens mit einer myocardial fixierten Electrode. Thoraxchirurgie, 20, 440-443.

Iwa, T., and Wada, J. (1974). Treatment of tachycardia by atrial pacing. Fapanese Circulation fournal, 38, 82-86.

Kahn, A. R., Morris, J. J., and Citron, P. (1976). Patientinitiated rapid atrial pacing to manage supraventricular tachycardia. American fournal of Cardiology, 38, 200-204.

Karlöf, I. (1975). Haemodynamic effect of atrial triggered versus fixed rate pacing at rest and during exercise in complete heart block. Acta Medica Scandinavica, 197, 195-206.

Kastor, J. A., and Leinbach, R. C. (1973). Pacemakers and their arrhythmias. In Cardiac Pacing, pp. 241-280, ed. P. Samet. Grune and Stratton, New York.

Kleinert, M., and Bisping, H. J. (1977). Erste klinische Erfahrungen mit einer neven transvenös endocardialen Schraubelektrode in Vorhof-und Kammerposition. Zeitschrift für Kardiologie, 66, 454-458.
Kleinert, M., Bock, M., and Wilhemi, F. (1977). Clinical use of a new transvenous atrieal lad. American fournal of Cardiology, 40, 237-242.

Moss, A. J. (1975). Therapeutic uses of permanent pervenous atrial pacemakers: a review. Fournal of Electrocardiology, 8, 373-380.

Moss, A. J., and Davis, R. J. (1974). Brady-tachy syndrome. Progress in Cardiovascular Diseases, 16, 439-454

Moss, A. J., and Rivers, R. J. (1978). Atrial pacing from the coronary vein. Circulation, 57, 103-106

Narula, O. S. (1971). Atrioventricular conduction defects in patients with sinus bradycardia. Circulation, 44, 10961110.

Nathan, D. A., Center, S., Wu, C. Y., and Keller, W. (1963). An implantable synchronous pacemaker for the long term correction of complete heart block. American fournal of Cardiology, 11, 362-367.

Paeprer, J., Thormann, I., and Nasseri, M. (1977). Cardiac pacing in the sick sinus syndrome. In Cardiac Pacing, pp. 200-203, ed Y. Watanabe. Excerpta Medica, Amsterdam.

Parsonnet, V., Furman, S., and Smyth, N. P. D. (1974). Implantable cardiac pacemakers status report and resource guideline. Circulation, 50, A-21-A-34.

Patel, A. K., Yap, V. U., and Thomsen, J. H. (1977). Adverse effects of right ventricular pacing in a patient with aortic stenosis. Chest, 72, 103-105.

Rosen, K. M., Loeb, H. S., Sinno, M. Z., Rahimtoola, S. H., and Gunnar, R. M. (1971). Cardiac conduction in patients with symptomatic sinus node disease. Circulation, 43, 836-844.

Rosenkranz, K. A., and Schaldach, M. (1971). Transvenösendokardiale Vorhofsteurung von Schrittmachern. Deutsche medizinische Wochenschrift, 96, 680-686.

Samet, P., Bernstein, W. H., Nathan, D. A., and Lopez, A. (1965). Atrial contribution to cardiac output in complete heart block. American fournal of Cardiology, 16, 1-10.

Samet, P., Castillo, C., and Bernstein, W. H. (1966). Hemodynamic sequelae of atrial, ventricular and sequential atrioventricular pacing in cardiac patients. American Heart fournal, 72, 725-729.

Shaw, D. B., and Eraut, C. D. (1970). The prevalence and morbidity of heart block in Devon. British Medical fournal, 1, 144-147.

Smyth, N. P. D., Citron, P., Keshishian, J. M., Garcia, J. M., and Kelly, L. C. (1976). Permanent pervenous atrial sensing and pacing with a new J-shaped lead. Fournal of Thoracic and Cardiovascular Surgery, 72, 565-570.

Sutton, R., and Perrins, E. J. (1979). To be published.

Sutton, R., Barrett, P., and Emanuel, R. (1978). In To Pace or Not to Pace-Controversial Subjects in Cardiac Pacing, pp. 324-329, ed H. J. Th. Thalen and J. W Harthorne. Nijhoff, Medical Division, The Hague, Netherlands.

Vera, Z., Mason, D. T., Awan, N. A., Miller, R. R., Janzen, D., Tonkon, M. J., and Vismara, L. A. (1977). Improvement of symptoms in patients with sick sinus syndrome by spontaneous development of stable atrial fibrillation. British Heart fournal, 39, 106-167.

Waxman, M. B., and Wald, R. W. (1977). Recurrent paroxysmal supraventricular tachycardia: a complication of ventricular pacing in a patient with occult Wolff-ParkinsonWhite syndrome. Fournal of Electrocardiology, 10, 291-298.

Waxman, M. B., Wald, R. W., Bonet, J. F., Macgregor, D. C., and Goldman, B. S. (1978). Self-conversion of supraventricular tachycardia by rapid atrial pacing. Pace, 1, 35-48.

Requests for reprints to Dr Richard Sutton, Westminster Hospital, Horseferry Road, London SW1. 